\title{
POSTER: Desenvolvimento de um Ambiente Virtual Imersivo para Auxiliar em Práticas de Falar em Público
}

\author{
$1^{\text {st }}$ C. G. S. Stedile \\ Unidade Acadêmica de Ciências Exatas \\ Universidade Federal de Goias \\ Jatai, Brasil \\ cgsste@hotmail.com
}

\author{
$2^{\text {nd }}$ G. S. Fleury \\ Unidade Acadêmica de Ciências Exatas \\ Universidade Federal de Goias \\ Jatai, Brasil \\ gabrielfleuri@hotmail.com
}

\author{
$3^{\text {rd }}$ M. W. S. Ribeiro \\ Unidade Acadêmica de Ciências Exatas \\ Universidade Federal de Goias \\ Jatai, Brasil \\ marcos_wagner@yahoo.com.br
}

\begin{abstract}
Este artigo apresenta um Ambiente Virtual Imersivo para auxiliar no processo de ensino em conteúdos de oratória. A partir de uma problemática estabelecida nas dificuldades de falar em público e nos resultados apurados nas avaliações formais em disciplinas com o conteúdo de oratória ou exposição, propõese um ambiente que se estabelece um ambiente simulado em que o usuário possa experimentar os variados contextos da fala em público. Os resultados obtidos por meio de avaliações (System Usability Scale(SUS) e Goal/Question/Metric(GQM)) permitiram demonstrar a validação da ferramenta construída e sua efetividade no alcance dos objetivos propostos.

Index Terms-RV, medo de falar em público, terapia
\end{abstract}

\section{INTRODUÇÃO}

O medo de falar em público (MFP) é geralmente tido como um dos piores medos da população, mesmo que este não seja resultado de uma situação de risco de vida. O MFP é uma forma comum de fobia social: cerca de um terço da população em geral relata ansiedade excessiva quando falam para um número relativo de pessoas, e, um terço deste grupo relata algum sofrimento ou prejuízo clinicamente significativo devido ao seu medo [8] [2] [3] [6] [11].

Existem várias intervenções possíveis para realizar o tratamento do MFP, a que mais se destaca é a terapia cognitivocomportamental (TCC) [13] [6]. Em conjunto com a TCC, a terapia de exposição (sistemática e paulatina - com estímulos que geram o medo em um ambiente controlado) é um tratamento bem estabelecido e eficaz de transtornos de ansiedade [9] [11].

A exposição in-vivo não é uma pratica comum, principalmente por ser onerosa, demorada e difícil de controlar variáveis externas [10]. Estas desvantagens podem ser superadas utilizando um meio alternativo para exposição: simulação usando meios computacionais tendo como premissa o realismo, o envolvimento e a interação. Estas definições, que se mostram como solução, são encontradas em uma única área, a realidade virtual $(\mathrm{RV})$.

A relevância desta combinação (RV e TCC) é bem demonstrada pela literatura como mostra [11]. E, a utilização da RV em conjunto com a técnica de exposição como forma de terapia tem sido empregada, com resultados positivos, ao tratamento de aracnofobia, agorafobia e medo de voar [7] [4] [12].Pesquisas utilizando a RV com exposição para tratamento de MFP mostraram-se tão efetivas quanto a exposição in-vivo [1] [6] [15] [11].

Atualmente, a maioria das pesquisas desenvolvidas para tratar o MFP com RV são muito estáticas (um único cenário para exposição, sem interação com a plateia) ou não aproveitam todo potencial da RV no quesito de controlar suas variáveis e/ou possibilidade de um ambiente dinâmico. A recente disponibilidade de equipamentos de RV para dispositivos móveis e o declínio drástico do custo provê um ambiente favorável para a adoção de novas maneiras de realizar a exposição por terapeutas. $\mathrm{O}$ desenvolvimento de um ambiente virtual altamente controlável, o qual possui plateia interativa, coleta de informações, retorno tanto para o usuário quanto para o psicólogo e a apresentação de técnicas para melhorar o desempenho pode aprimorar a qualidade do tratamento realizado.

Portanto a criação de uma ferramenta que permite tais possibilidades descritas anteriormente ao psicologo é imperativa. Desta forma, o objetivo deste trabalho é analisar se a utilização de uma ferramenta com RV imersiva para realizar tratamentos de pessoas com medo de falar em público é valida neste contexto. Para atingir este objetivo geral, foram definidos os seguintes objetivos específicos: a) O primeiro destes objetivos é a criação de diversos cenários diferentes para ser realizado o tratamento. b) $\mathrm{O}$ segundo é a possibilidade de interação em tempo real da plateia com o orador de acordo com a vontade do psicólogo, tendo o público da disciplina citada como primeira experimentação. c) E o terceiro objetivo é a coleta de informações em tempo real do paciente por meio de sensores cardíacos e galvânicos; assim como a coleta de informações de onde o paciente mais olha no ambiente virtual e a gravação do discurso feito pelo paciente.

\section{ARquitetura E IMPLEMENTAÇÃo}

Com as propostas feitas anteriormente em mente, foi desenvolvido um ambiente virtual o qual possui uma plateia 
dinâmica. $\mathrm{O}$ ambiente virtual criado foi remetido a um auditório(Ver figura 1) em que o usuário do sistema em RV faz uma palestra. A plateia é controlada por meio de um módulo o qual controla o ânimo (interessada, normal e desinteressada) e a quantidade de pessoas na sala.

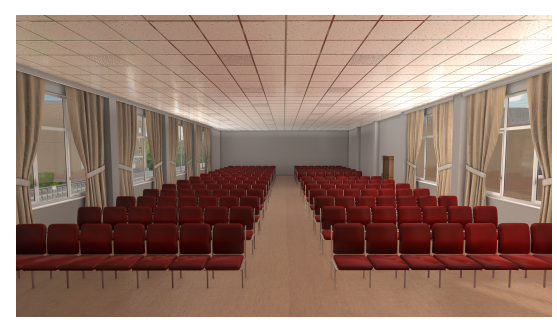

Fig. 1. Auditório o qual o usuário irá realizar a apresentação

O ambiente virtual foi desenvolvido com o uso do motor de jogos Unity, o qual permitiu a criação e ordenação dos objetos 3D e imersão do usuário. Para a codificação feita foi utilizada a linguagem de programação C\#. Antes de entrar no ambiente virtual, o usuário é informado sobre a necessidade de fazer um discurso sobre um assunto por cinco minutos.

Dentro do ambiente virtual, o usuário pode selecionar quantas pessoas estarão na plateia, por meio de botões virtuais no próprio cenário, os quais ficam na parede atrás do usuário. $\mathrm{O}$ usuário também pode selecionar qual o grau de interesse da plateia presente, se estão bastante interessados, meio interessados, pouco interessados ou nada interessados.

Por conta da dificuldade de se gerar uma vasta quantidade diferente de pessoas virtuais, foram utilizados modificações das mesmas, como mudança de cor da roupa, cor do cabelo e posição da pessoa.

Dentre as opções providas anteriormente, o usuário será o centro da atenção durante os seus cinco minutos de fala. Caso a plateia esteja selecionada para ter o máximo de atenção, todos estarão olhando para o usuário do sistema com interesse, querendo ouvir mais do que o usuário está falando.Caso seja selecionado o mínimo de atenção, nenhuma, para a plateia, os ouvintes não prestarão atenção no usuário, conversarão entre si e farão barulho para atrapalhar a apresentação.

Para o usuário não se sentir mal no final da sua apresentação, todos da plateia batem palmas.

\section{AnÁlise E TESTES}

Para realizar a avaliação deste AVI, foi utilizado um dos mais conhecidos e mais simples métodos de averiguação do nível de usabilidade de um sistema, denominado: System Usability Scale (SUS), proposto por Brooke et al., 1996. O SUS pode ser utilizado para avaliar aplicação, software, websites, serviços, hardware e qualquer outro tipo de interface. O SUS permite avaliar os seguintes critérios: a) Efetividade (os usuários conseguem completar seus objetivos?) b) Eficiência (quanto esforço e recursos são necessários para isso?) c) Satisfação (a experiência foi satisfatória?)

\section{Conclusões e Trabalhos Futuros}

$\mathrm{O}$ intuito desta pesquisa (produto final) foi apresentar uma ferramenta auxiliar no processo de prática de falar em público motivado pelos resultados obtidos em uma disciplina de Trabalho de Conclusão de Curso em que o conteúdo oratória era trabalhado. Com esta intenção o primeiro passo foi a validação da proposta, que apesar de estar apresentada resumidamente na seção anterior (Análise e Testes) foi alcançada. Apesar de ser utilizada como meio de prática a ferramenta tem um potencial que extrapola os limites da simples prática indo ao encontro de questões relacionados ao trauma e ao psíquico das pessoas.

\section{REFERENCES}

[1] A. Felnhofer, J. Kafka, H. Hlavacs, L. Beutl, I. Kryspin-Exner and O D. Kothgassner. "When you meet others in an everyday interpersonal situation virtually: Investigating prosocial behavior and social avoidance towards avatars and agents", Computers in Human Behavior, vol. 80, Nov. 2017

[2] A. M. Ruscio, T. Brown, W. Tat Chiu, J. Sareen, M. B. Stein and R. Kessler. "Social fears and social phobia in the usa: Results from the national comorbidity survey replication.", Psychological medi cine, vol. 38 , pp. $15-28$, Jan. 2008

[3] American Psychiatric Association, Diagnostic and statistical manual of mental disorders: DSM-5, 5th ed., 2013

[4] E. Malbos, R. M. Rapee and M. Kavakli. "A controlled study of agoraphobia and the independent effect of virtual reality exposure therapy", The Australian and New Zealand journal of psychiatry, vol. 47, pp. 160-168, July 2012.

[5] E. Mayo-Wilson, S. Dias, I. Mavranezouli, K. Kew, D. Clark, A. Ades and S. Pilling. "Psychological and pharmacological interventions for social anxiety disorder in adults: A systematic review and network metaanalysis.", The Lancet Psychiatry, vol. 1, pp.368-376, Oct. 2014

[6] H. E. Kim, Y.-J. Hong, M.-K Kim, Y. H. Jung, S. Kyeong, and J.-J. Kim. (2017). "Effectiveness of self-training using the mobile-based virtual reality program in patients with social anxiety disorder", Computers in Human Behavior, vol. 73, pp. 614-619, Aug. 2017

[7] J. Almeida, D. Suarez, F. Tapia and G. Guerrero. "Use of virtual reality using render semi-realistic as an alternative medium for the treatment of phobias. case study: Arachnophobia", in First international Conference ICAI, Bogotá, Colombia, 2018, pp. 144-154.

[8] M. B. Stein, J. Walker and D. Forde. "Public-speaking fears in a community sample. prevalence, impact on functioning, and diagnostic classification.", Archives of general psychiatry, vol. 53, pp. 169-174, March 1996

[9] M. G. Craske, M. Treanor, C. Conway, T. Zbozinek and B. Vervliet. "Maximizing exposure therapy: An inhibitory learning approach", Behaviour research and therapy, vol 58, pp. 10-23, May 2014

[10] O. Weisman, I. Aderka, S. Marom, H. Hermesh and E. GilboaSchechtman, E. "Social rank and affiliation in social anxiety disorder", Behaviour research and therapy, vol. 49, pp. 399-405, June 2011

[11] P. Lindner, A. Miloff, S. Fagernas, J. Andersen, M. Sigeman, G. Andersson, T. Furmark and P. Carlbring. "Therapist-led and self-led one-session virtual reality exposure therapy for public speaking anxiety with consumer hardware and software: A randomized controlled trial", Journal of anxiety disorders, vol 61, pp.45-54, Jan. 2019

[12] R. A. Cardo, O. A. David and D. O. David. "Virtual reality exposure therapy in flight anxiety", Computers in Human Behavior, vol. 72, pp. 371-380, July 2017

[13] R. G. Heimberg, M. R. Liebowitz, D. A. Hope, F. R. Schneier, C. S. Holt, L. A. Welkowitz, H. R. Juster, R. Campeas, M. A. Bruch, M. Cloitre, B. Fallon and D. F. Klein. "Cognitive Behavioral Group Therapy vs Phenelzine Therapy for Social Phobia: 12-Week Outcome", Arch Gen Psychiatry, vol. 55, pp. 1133-1141, Dec. 1998

[14] V.R. Basili, G. Caldiera and H. D. Rombach. "The Goal Question Metric Approach", 1994

[15] Y. H. T. Perandre and V. B. Haydu. "Um Programa de Intervenção para Transtorno de Ansiedade Social com o Uso da Realidade Virtual", Trends in Psychology, vol. 26, pp. 851-866, 2018 